\section{Electrospun antimony doped tin oxide (ATO) nanofibers as a versatile conducting matrix $\dagger$}

\author{
Rainer Ostermann, ${ }^{a}$ Roman Zieba, ${ }^{b}$ Melanie Rudolph, ${ }^{c}$ Derck Schlettwein ${ }^{c}$ and \\ Bernd M. Smarsly*a
}

Received 22nd June 2011, Accepted 3rd October 2011

DOI: $10.1039 / \mathrm{clcc13724g}$

Nanoparticles of ATO (antimony doped tin oxide) were used to produce thick conductive, free standing mats of nanofibers via electrospinning. These fibrous mats were incorporated into polymer films to produce a transparent conducting polymer foil. Moreover, the fiber mats can serve as porous electrodes for electrodeposition of Prussian $\mathrm{Blue}$ and $\mathrm{TiO}_{2}$ and were tested in dye-sensitized solar cells.

TCO (transparent conducting oxide) materials are widely used in opto-electronic applications as transparent electrodes, commonly as thin, compact films deposited on glass or quartz substrates. ${ }^{1,2}$ These TCO layers of 200-300 nm thickness possess sheet resistances typically below $20 \Omega / \square$ and transparencies above $85 \%$ in the visible range, making them also suitable as two-dimensional electrodes for electrochromic devices ${ }^{3}$ and solar cells. ${ }^{4}$ TCO films with $3 \mathrm{D}$ porosity with pore sizes beyond $100 \mathrm{~nm}$ are supposed to be useful for diverse applications. As proposed by Zaban et al., a 3D TCO electrode theoretically offers advantages for electron collection in DSSCs (dye-sensitized solar cells), ${ }^{5}$ due to a higher surface area and a shorter electron diffusion pathway to the back electrode. The practical realization of such DSSCs with $\mathrm{TiO}_{2}$ on ITO (indium tin oxide) nanopowders ${ }^{6}$ and nanowires generated by laser-ablation ${ }^{7}$ showed only low conversion efficiency, but recently a more elaborate approach based on a poly(carbonate) template yielded around $4 \%$ efficiency. ${ }^{8}$ In addition, porous TCO electrodes might prove suitable in other applications with non-conducting polymers or oxides with low intrinsic conductivities, as for example oxide cathodes for Li-batteries. ${ }^{9}$

Here we demonstrate a novel procedure allowing the production of ATO (antimony doped tin oxide) nanofiber mats and their usage to endow intrinsically insulating polymer films with conductivity, and as highly porous electrodes for electrodeposition and device fabrication. To the best of our knowledge, this is the first working

\footnotetext{
${ }^{a}$ Justus Liebig University, Institute of Physical Chemistry,

Heinrich-Buff-Ring 58, D-35392 Giessen, Germany.

E-mail: bernd.smarsly@phys.chemie.uni-giessen.de;

Fax: + 49 6419934509; Tel: + 496419934590

${ }^{b}$ BASF SE, GVP/E, D-67056 Ludwigshafen, Germany

${ }^{c}$ Institut für Angewandte Physik, Heinrich-Buff-Ring 16,

D-35392 Giessen, Germany

$\dagger$ Electronic supplementary information (ESI) available. See DOI: $10.1039 / \mathrm{clcc} 13724 \mathrm{~g}$
}

example of a nanofiber-based ATO electrode. For ordinary TCO electrodes, typical deposition techniques are CVD and PVD (chemical and physical vapor deposition), including spray pyrolysis, PLD (pulsed laser deposition) and magnetron sputtering, resulting in compact layers. Various attempts have been made to employ sol-gel processing, yielding good transparency, but generally much lower conductivity. Main benefits of sol-gel approaches are the possibility of solution-based coating technology and the facile generation of porosity through suitable polymer templates, as demonstrated for mesoporous and macroporous ITO. ${ }^{10,11}$ An alternative concept to endow TCOs with porosity is the generation of nanofibers by electrospinning. The combination of a suitable polymer as a carrier with conventional sol-gel processing thus can be applied for the electrospinning of metal oxide fibers, including ITO. ${ }^{8,15}$ However, for other n-type TCO materials like ATO, FTO (fluorine doped tin oxide) or AZO (aluminum doped zinc oxide) only low conductivities have been reported, even for nonporous films, while these TCOs are desirable substitutes of the costly ITO. ${ }^{12}$ In contrast to ITO, for these ternary oxides a sol-gel route leads to a non-ideal stoichiometry and a phase-separation into the two corresponding oxides, thus resulting in a lower conductivity. These shortcomings can be overcome to some extent by using preformed nanoparticles. ${ }^{13,14}$

In this work we extend the electrospinning of TCO nanofibers by using a dispersion of colloidal ATO nanoparticles instead of classical sol-gel precursors to obtain more stable electrospinning solutions. Further advantages of this approach are the control of stoichiometry and crystallinity of the preformed nanoparticles, which are especially beneficial for ternary oxides like TCOs. A main challenge was to develop ATO nanoparticle dispersions enabling electrospinning of high-quality nanofibers. A precipitation method from the literature ${ }^{13}$ was modified to create highly crystalline ATO nanoparticles (Fig. S1 and S2, ESI $\dagger$ ) of ca. 3-6 nm in size (Fig. S2 and S3, ESI $\dagger$ ) that did not contain ionic stabilizers and could thus be redispersed in solvents suitable for electrospinning. In a typical electrospinning experiment, a dispersion of ATO nanoparticles (20 wt \%) was spun with PVP (poly(vinyl pyrrolidone) as a carrier polymer (see ESI $\dagger$ for details) to produce a non-woven mat.

As an important step to increase the packing density of the fibers, this mat was soaked with hexane and dried at room temperature, before calcination at $550{ }^{\circ} \mathrm{C}$ for $10-20 \mathrm{~min}$. 
The free-standing fiber mat could then be fixed onto a glass or commercial FTO (Haller Glas, Germany) substrate by wetting the substrate with a drop of $5 \mathrm{wt} \% \mathrm{Sn}(\mathrm{IV}) \mathrm{Cl}_{4}$ solution in ethanol. This procedure might seem cumbersome, but taking into account the $15-20 \%$ shrinkage of the fibers during calcination, it is the only way to avoid extensive rupture and delamination of the nanofibers, as it occurs for nonfreestanding mats on substrates. We believe this problem to be the main reason that TCO nanofiber mats have not been used so far for electrodeposition or device fabrication (other than single fiber sensing), although there are several reports on ITO nanofibers, ${ }^{16}$ showing good conductivity for an individual nanofiber. Actually, the sol-gel approach in these works leads to even more pronounced shrinkage, but our fixation procedure is equally applicable for such sol-gel derived nanofiber mats, while we only found the sol-gel derived ITO fibers to be highly conductive. For other TCO materials including ATO, the nanoparticle approach seems more viable.

To evaluate the macroscopic conductivity, the resistance of fibers mats on glass was measured over a distance of $1 \mathrm{~cm}$ between contacts made from conducting silver paste. For a mat electrospun for $120 \mathrm{~s}$ typically the resistance is approx. $400 \Omega \mathrm{cm}^{-1}$ (see Fig. S4, ESI $\dagger$ ), being only one order of magnitude higher than for commercial TCO-coated glass. The nanofiber mats could be generated with variable thickness (controlled by the spinning time), all possessing reasonable conductivities. This was demonstrated by depositing Prussian Blue (Iron(II,III) hexacyanoferrate). ${ }^{17}$

As shown in Fig. 1, deeply colored films were obtained, and Prussian Blue was homogeneously deposited onto the fibers. In an aqueous solution of $0.1 \mathrm{M} \mathrm{KCl}$ as an electrolyte, the cyclovoltammogram shows oxidation and reduction peaks at the same potentials as for a Prussian Blue film deposited on commercial FTO glass (see Fig. S5, ESI $\dagger$ ). Similarly, the electrochromic behavior of Prussian Blue, i.e. the reversible coloration and bleaching, was observable with a stable response for at least ten cycles. Whereas the electrodeposition of Prussian Blue on ATO nanofibers proves the applicability of the methodology, the concept of fibrous electrodes is more universal and offers advantages in other systems like $\mathrm{TiO}_{2}$-based dye-sensitized electrodes. $\mathrm{TiO}_{2}$ can be anodically deposited from acid $\mathrm{TiCl}_{3}$ solution and the addition of surfactants

\section{Electrospinning}

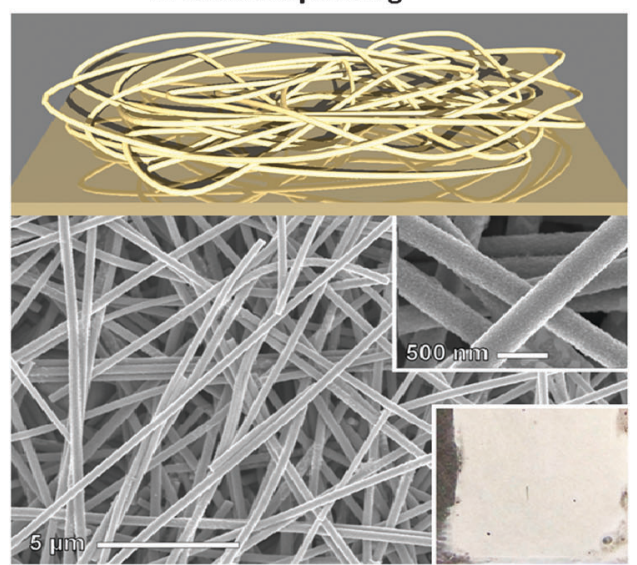

\section{Electrodeposition of Prussian Blue}

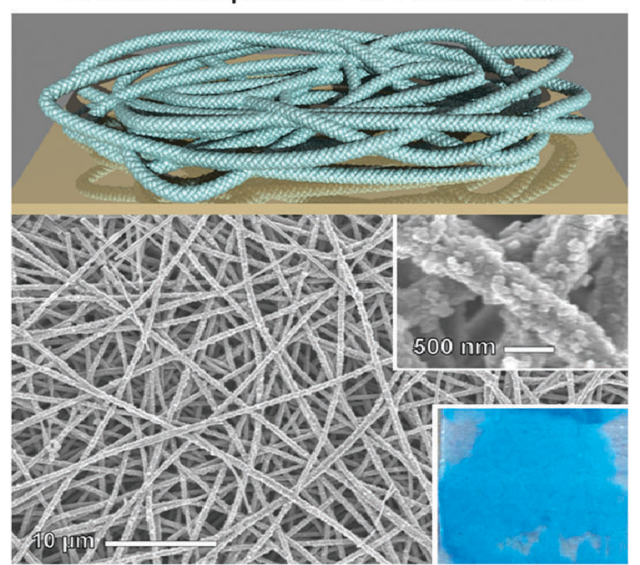

Fig. 1 ATO nanofiber electrode before (left) and after (right) Prussian Blue electrodeposition: scheme (top), SEMs and digital photographs (lower inset) of the fiber mat used as an electrode on a glass slide.
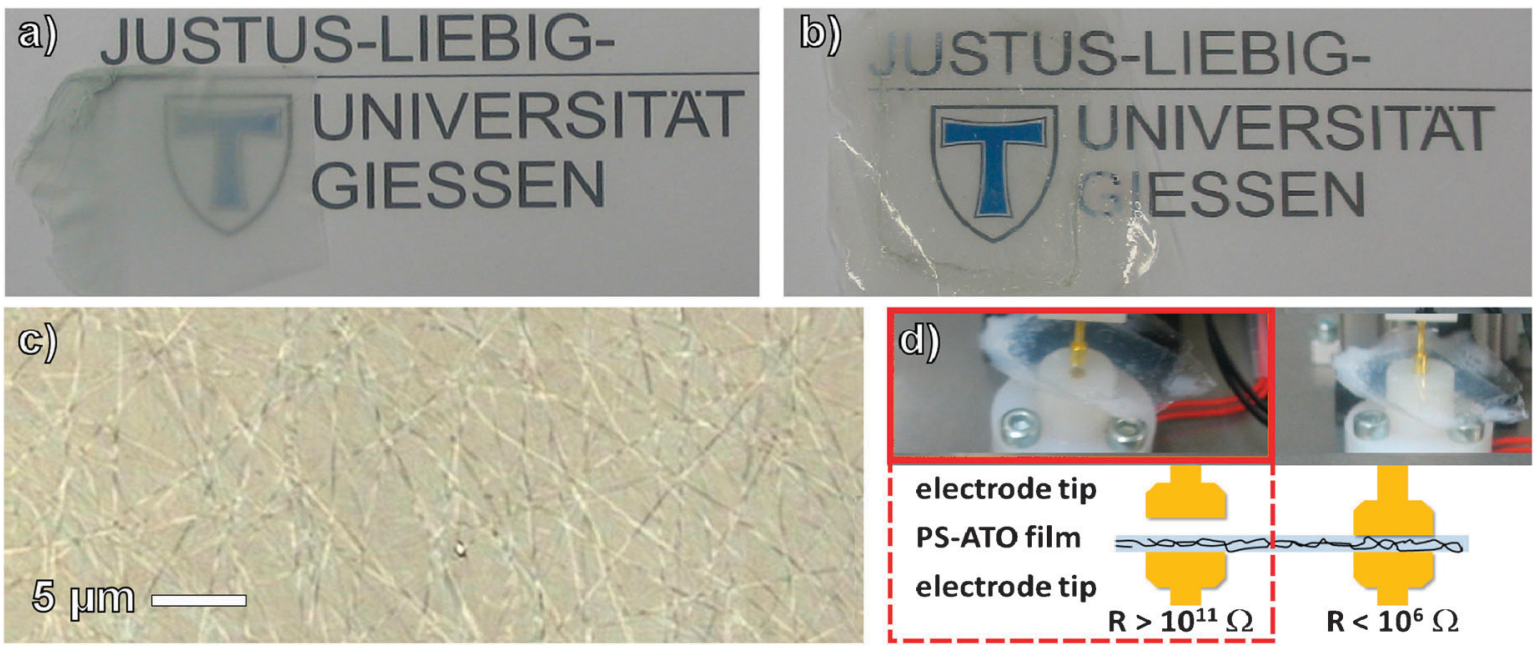

Fig. 2 Digital photographs of ATO nanofiber mats (a) in air and (b) inside poly(styrene). The latter composite poly(styrene) (PS) film containing the ATO nanofibers imaged with a laser microscope (c) and the macroscopic resistivity through the PS-ATO film (d). 
like SDS (sodium dodecylsulfate) or BTB (bromothymol blue) affords the generation of porous titania. ${ }^{18,19}$ However, when the thickness of the titania layer exceeds $500 \mathrm{~nm}$ on flat substrates cracks appear and the film tends to peel off, thus severely limiting light absorption and the solar cell efficiency (see Fig. S6, ESI $\dagger$ ). In contrast, a deposit of $500 \mathrm{~nm}$ of $\mathrm{TiO}_{2}$ on each nanofiber in a thick fiber mat results in an overall thickness of several microns (see Fig. S7, ESI $\dagger$ ). Consequently, the dye-sensitized solar cells based on our nanofibrous ATO electrodes generate a higher photocurrent of $3.9 \mathrm{~mA} \mathrm{~cm} \mathrm{~m}^{-2}$ than solar cells similarly prepared on flat FTO substrates showing a value of $0.9 \mathrm{~mA} \mathrm{~cm}^{-2}$ (Fig. S8, ESI $\dagger$ ). Although the cell displayed a fill factor of only $33 \%$, possibly due to defects in the blocking layer on the fibers, we observed conversion efficiencies of up to $1 \%$ for a standard Graetzel-cell test without optimization (other than using a flat FTO substrate instead of a glass slide as back contact to avoid a high internal resistance).

Another field of application for the ATO nanofibers mats can be found in polymer films that are usually insulating. We demonstrated a hybrid polymer-TCO film with antistatic properties and good transparency (unlike CNT hybrids ${ }^{20}$ ) by simply hot pressing our ATO nanofiber mats into poly(styrene) or poly(vinylpyrrolidone) (Fig. 2, see ESI $\dagger$ for the synthesis). Since the average indices of refraction of the polymer and the ATO fibers were similar, the hybrid films showed high transparency, in contrast to the nanofiber mats themselves. The resistivity was determined by 2-probe measurement to be $20 \mathrm{k} \Omega \mathrm{cm}^{-1}$ in-plane and $100-300 \mathrm{k} \Omega$ perpendicular to the composite film. As seen by a laser microscope (Fig. 2), the fibers were homogeneously distributed within the polymeric matrix. The optical transmission was ca. $75 \%$ (at $500 \mathrm{~nm}$ ). Thus, high conductivity can be achieved at moderate loadings of ATO fibers owing to a quite low percolation threshold. Such hybrids take advantage of the nanofibers' conductivity while offsetting their fragility and therefore represent an interesting type of antistatic and transparent polymer film. Such conducting polymer hybrids can also be achieved with carbon nanotubes, ${ }^{20}$ but evidently the transparency is a significant advantage of the TCO alternative.

In conclusion, we demonstrated a novel approach for the fabrication of nanostructured electrodes based on electrospun
ATO nanofibers and their use as porous electrodes and for the generation of conducting composite films with insulating polymers of high industrial impact. We are further convinced that our approach of generating and using TCO fiber mats represents a novel conceptual methodology with substantial potential in diverse fields requiring a three-dimensional electrode.

\section{Notes and references}

1 T. Minami, Semicond. Sci. Technol., 2005, 20, S35-S44.

2 P. P. Edwards, A. Porch, M. O. Jones, D. V. Morgan and R. M. Perks, Dalton Trans., 2004, 2995-3002.

3 C. G. Granqvist, Appl. Phys. A: Solids Surf., 1993, 57, 19-24.

4 E. Fortunato, D. Ginley, H. Hosono and D. C. Paine, MRS Bull., 2007, 32, 242-247.

5 S. Chappel, L. Grinis, A. Ofir and A. Zaban, J. Phys. Chem. B, 2005, 109, 1643-1647.

6 L. Grinis, A. Ofir, S. Dor, S. Yahav and A. Zaban, Isr. J. Chem., 2008, 48, 269-275.

7 E. Joanni, R. Savu, M. de S. Góes, P. R. Bueno, J. N. de Freitas, A. F. Nogueira, E. Longo and J. A. Varela, Scr. Mater., 2007, 57 $277-280$

8 H.-W. Wang, C.-F. Ting, M.-K. Hung, C.-H. Chiou, Y.-L. Liu, Z. Liu, K. R. Ratinac and S. P. Ringer, Nanotechnology, 2009, 20, 055601 .

9 M. S. Whittingham, Chem. Rev., 2004, 104, 4271-4302.

10 D. Fattakhova-Rohlfing, T. Brezesinski, J. Rathousky, A. Feldhoff, T. Oekermann, M. Wark and B. M. Smarsly, $A d v$. Mater., 2006, 18, 2980-2983.

11 X. Zhang, Y. Man, J. Wang, C. Liu and W. Wu, Sci. China, Ser. E. Technol. Sci., 2006, 49, 537-546.

12 E. Shanthi, V. Dutta, A. Banerjee and K. L. Chopra, J. Appl. Phys., 1980, 51, 6243-6251.

13 C. Goebbert, R. Nonninger, M. A. Aegerter and H. Schmidt, Thin Solid Films, 1999, 351, 79-84.

14 M. M. Munir, F. Iskandar, K. M. Yun, K. Okuyama and M. Abdullah, Nanotechnology, 2008, 19, 145603.

15 D. Li and Y. Xia, Adv. Mater., 2004, 16, 1151-1170.

16 D. Lin, H. Wu, R. Zhang and W. Pan, Nanotechnology, 2007, 18, 465301 .

17 J. J. Garcia-Jareno, D. Benito, J. Navarro-Laboulais and F. Vicente, J. Chem. Educ., 1998, 75, 881-884.

18 L. Kavan, B. O'Regan, A. Kay and M. Graetzel, J. Electroanal. Chem., 1993, 346, 291-307.

19 K. Wessels, A. Feldhoff, M. Wark, J. Rathousky and T. Oekermann, Electrochem. Solid-State Lett., 2006, 9, C93-C96.

20 J. K. W. Sandler, J. E. Kirk, I. A. Kinloch, M. S. P. Shaffer and A. H. Windle, Polymer, 2003, 44, 5893-5899. 